\title{
An Efficient Associative Processor Solution to an Air Traffic Control Problem
}

\author{
Mike Yuan, Johnnie Baker \\ Department of Computer Science \\ Kent State University \\ Kent, Ohio 44242 \\ Email: myuan, jbaker@cs.kent.edu
}

\author{
Frank Drews, Lev Neiman \\ School of Electrical Engineering \\ and Computer Science \\ Ohio University \\ Athens, $\mathrm{OH}$
}

\author{
Will Meilander(retired) \\ Department of Computer Science \\ Kent State University \\ Kent, $\mathrm{OH}$ \\ Email:willcm@charter.net
}

Email:drews@ohio.edu,lev.neiman@gmail.com

\begin{abstract}
This paper proposes a SIMD solution to air traffic control (ATC) using an enhanced SIMD machine model called an Associative Processor (AP). This differs from previous ATC systems that are designed for MIMD computers and have a great deal of difficulty meeting the predictability requirements for ATC, which are critical for meeting the strict certification standards required for safety critical software components. The proposed SIMD solution will support accurate and meaningful predictions of worst case execution times and will guarantee all deadlines are met. Also, the software will be much simpler and smaller in size than the current corresponding ATC software. An important consequence of these features is that the $V \& V$ (Validation and Verification) process will be considerably simpler than for current ATC software. Additionally, the associative processor is enhanced SIMD hardware and is considerably cheaper and simpler than the MIMD hardware currently used to support ATC. The ClearSpeed CSX600 accelerator is used to emulate the AP model. A preliminary implementation of the proposed method has been developed and experimental results comparing MIMD and CSX600 approaches are presented. The performance of CSX600 has better scalability, efficiency, and predictability than that of MIMD.
\end{abstract}

Keywords-Air Traffic Control; ATC; Air Traffic Management; Conflict Detection and Resolution; CD\&R; Associative Processor; AP; ClearSpeed CSX600; SIMD; MIMD;

\section{INTRODUCTION}

The Air Traffic Control (ATC) system is a real-time system that continuously monitors, examines, and manages space conditions for thousands of flights by processing large volumes of data that are dynamically changing due to reports by sensors, pilots, and controllers, and gives the best estimate of position, speed and heading of every aircraft in the environment at all times. The ATC software consists of multiple real-time tasks that must be completed in time to meet their individual deadlines. The FAA has spent a great deal of effort on finding a predictable and reliable system to achieve free flight which would allow pilots to choose the best path to minimize fuel consumption and time delay rather than following pre-selected flight corridors [12], [22], [29]. Massive efforts have been devoted to finding an efficient MIMD solution to the ATC problems for many years. Previous attempts to build a new system have repeatedly failed after about ten years work by a large team [20], [21].

The most critical issue of free flight is conflict detection and resolution. The performance of all CD\&R algorithms available depends on aircraft state estimation according to the comprehensive survey of Kuchar and Yang[14]. The Kalman filter[1], [4] is the central algorithm for the majority of all modern tracking systems, known as $\alpha-\beta, \alpha-\beta-\gamma$ filters. The major problem with the single Kalman filter is that it does not predict well when the aircraft makes an unanticipated change of flight mode such as making a maneuver, accelerating etc [17]. Many adaptive state estimation algorithms have been proposed [18], [15], [2], [28]. The Interacting Multiple Model (IMM) algorithm [3], [16] runs two or more Kalman filters that are matched to different modes of the system in parallel. It uses a weighted sum of the estimates from the bank of Kalman filters to compute the state estimate. IMM and its variants have been applied to single and multiple aircraft tracking problems in [18]. However, it becomes inaccurate for tracking multiple aircraft as the number of aircraft increases. Current MIMD implementation of this algorithm is very computational intensive. Hwang et. al. [9] propose that the mode likelihood function can be used to improve the estimation results of IMM algorithm. The likelihood function uses the mean of the residual produced by each Kalman filter.

A comprehensive survey of the CD\&R algorithms is presented in Kuchar and Yang [14]. In [13], Krozel et. al. propose one centralized strategy that is controller-oriented and two decentralized strategies that are user-oriented. In the centralized approach, a central agent analyzes the trajectories of the aircraft and determines resolutions. In the two decentralized strategies, each aircraft resolves its own conflicts as they are detected. In [29], Yang et. al. propose a conflict alerting logic based on sensor and trajectory uncertainties, with conflict probability based on Monte Carlo simulation. Chiang et. al. [5] propose CD\&R algorithms from the perspective of computational geometry. Paielli et. al. [23] and Prandini et. al. [25] propose analytic algorithms for computing probability of conflict. Hwang et. al. [9] use their residual mean IMM tracking algorithm to detect conflicts. Many of the algorithms consider only two aircraft. 
For example, Krozel et. al. [13] show that neither their centralized nor decentralized CD\&R algorithms can guarantee safety for multiple aircraft when the number of aircraft is growing. Furthermore, many algorithms propose optimization schemes that are not guaranteed to be completed within real-time deadlines. Due to the increasing number of FAA problems, FAA is inviting proposals for new and efficient CD\&R proposal [32].

On the other hand, several papers [19], [20], [21], [30] have used an Associative Processor (AP) to manage ATC computation. The AP assumed an enhanced SIMD model which can execute several global operations in constant time, as will be explained in detail in Section III. The assumed maximum number of aircraft being tracked by one air traffic control center is 4000 IFR (instrument flight rules) aircraft and 10000 VFR (visual flight rules) aircraft, for a total of 14000 aircraft [19], [20]. This involves maintaining flight information for all flights in the region controlled by one air traffic control region plus all flights that are in the other regions that are adjacent to this region. There are 20 flight control centers in the lower (or contiguous) U.S. states plus one each for Hawaii and Alaska.

An AP for ATC will have sufficient parallel processor memory so that all the records for each aircraft can be stored permanently in the memory of a single processor, as the movement of very large sets of records is very time consuming. The memory size and speed for the parallel processors in a SIMD with upward tens of thousands of processors is typically small due to cost issues, thereby restricting the number of aircraft that each processor can manage. Also, if the maximum number of aircraft assigned to a single processor is large, this also severely impacts the AP's performance. For example, if a maximum of 100 aircraft are assigned to one processor, then the running time is increased by a factor of more than 100 over the running time achieved if each processor is assigned at most one aircraft.

A primary reason that an AP is ideal for the ATC problem is the speedup that it can achieve. If an AP has $n$ processors, it can execute simultaneously $n$ instances of the same tasks in essentially the same time that it requires to provide one instance of this task, which allows the AP to achieve nearoptimal speedup. Although, assigning multiple aircraft to each processor is certainly feasible, the assignment of at most one aircraft to each processor is ideal. This will allow the maximum number of different ATC tasks to be processed and meet the individual deadlines for each task.

A second reason that the AP is ideal for ATC is that it has already been shown that the AP can handle this problem [19], [20], [21]. In fact, the STARAN AP was designed by Kenneth Batcher and built by Goodyear Areospace explicitly for ATC. The ASPRO AP was a second generation STARAN and numerous units were purchased by the NAVY and used for the related problem of air defense systems. These
APs were considered to be natural database machines and intended for dynamic database applications like ATC. The deterministic architecture of a SIMD supported extremely accurate worst case estimates of running time, allowing a static schedule to be used instead of dynamic scheduling. Data is moved deterministically by use of broadcast or interconnection network algorithms, so that it is always available when it is needed. The associative properties supported constant time execution of standard database activities [11]. As discussed in [20], [21], many time-consuming activities like dynamic scheduling, load balancing, shared resource management, assuring ACID properties for database transactions are either simple to handle or activities that are not needed for SIMD, due largely to its single instruction stream. That SIMD is a very different type of parallel machine is demonstrated by the fact that the proofs of most or all of the well-known NP-hard problems involving multiprocessors [6] do not apply to SIMDs and, in fact, software solutions or approximate solutions to these types of problems are not needed in solutions of other problems.

Since the number of aircraft continues to increase rapidly and there is a preference at FAA to consolidate similar activities to minimize aircraft handoffs when possible and to perform multiple backup computations for redundancy purposes (e.g., for nearby regions), it is realistic to expect that ATC could fully utilize an AP with $100 k$ or more PEs in the implementation of its current NextGen project.

In this paper, the ClearSpeed CSX600 is used to emulate the AP solution to the ATC problem. Our ATC algorithms use CSX600 SIMD features to process up to 96 instances of the same task synchronously. The architecture of our proposed AP is much smaller and simpler than previous and current ATC MIMD architectures. Our preliminary experimental results in Section V compare the performance and predictability of CSX600 implementation and two MIMD implementations of aircraft tracking algorithm.

This paper is organized as follows. Section II overviews CSX600 architecture and programming concepts. We discuss emulating the AP on the CSX600 in Section III. Section IV presents our approaches for key ATC tasks such as aircraft tracking, conflict detection and resolution etc. Section V presents experimental results. Conclusions and future work are presented in Section VI.

\section{OVERVIEW OF CleARSPEEd CSX600}

The ClearSpeed accelerator board shown in Figure 1 is a PCI-X card equipped with two CSX600 coprocessors. The CSX600 board is a multi-core processor with two CSX600 coprocessors, each with 96 processing elements (PEs) connected in the form of a one-dimensional array. At present, we are only using one of the two coprocessors in order to obtain a more SIMD-like environment. This multi-core section is called a multi-threaded array processor (MTAP) core, and the architecture is shown in Figure 2. The 


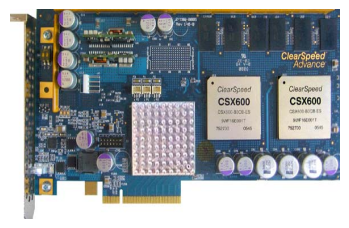

Figure 1: CSX600 accelerator board

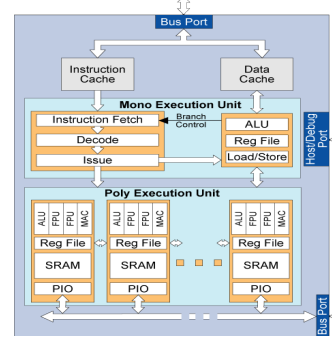

Figure 2: MTAP architecture of CSX600

programmer only has to provide a single instruction stream, and the instructions and data are dispatched to the execution units that have two parts: one is mono that functions as a control unit and processes non-parallel data, and the other is poly that has 96 PEs. At each step, all active PEs execute the same command synchronously on their individual data. Each PE has its own local memory of 6 Kbytes, a dual 64bit FPU, its own ALU, integer MAC, registers and I/O. The PEs operate at a clock speed of $250 \mathrm{MHz}$. The aggregate bandwidth of all PEs is specified to be 96 Gbytes/s, which is for on-chip memory. Further information on the hardware architecture can be found in the documentation [33].

The ClearSpeed accelerator provides the $C^{n}$ language as the programming interface for the CSX600 processors. It is very similar to the standard $\mathrm{C}$ programming language. The main difference is that it introduces two types of variables, namely mono and poly variables. The mono variables are equivalent to common $\mathrm{C}$ variables and used by the control unit. The poly values have an instance on each PE and are processed by the PEs. Further information on the associated software can be found in the documentation [34], [35]. There are three library functions for data transfer on the ClearSpeed. The first one is from mono to poly meтсруm $2 p$, second one is from poly to mono memcрyp $2 m$, and third one is to exchange data with adjacent PEs using the swazzle network, which is a ring network connecting all the processors together. More details of usages of library functions can be found in the documentation [35], [7].

\section{Emulating the AP on the ClearSpeed CSX600}

An Associative Processor (AP) [19] is a SIMD machine with additional hardware enhancements. A more complete
Table I: Timings of Associative Functions

\begin{tabular}{|c|c|c|}
\hline Associative functions & Timings in $C^{n}$ & Timings in assembly \\
\hline max & 5.257 & 3.654 \\
\hline min & 5.257 & 3.654 \\
\hline AND & 7.024 & NA \\
\hline OR & 7.364 & NA \\
\hline associative search & 29.2 & NA \\
\hline any & 0.281 & NA \\
\hline nany & 15.147 & 8.245 \\
\hline get & 13.116 & 7.876 \\
\hline next & 13.032 & 7.816 \\
\hline broadcast & 100 & NA
\end{tabular}

and careful listing of the associative properties follows [11], [24]:

- MAX and MIN: Global reduction operations of integers or real numbers that occur in each instance of the same record across all active PEs using maximum or minimum.

- AND and OR: Global reduction operations of Boolean values in each instance of the same record across all active PEs using AND or OR.

- Associative search: Finds all instances of the same record across all active PEs whose data values match the search pattern. The active PEs whose data value in the record matches the search pattern are called responders, and the active PEs whose data value in the record does not match the search pattern are called nonresponders.

- Any-Responders: ANY is to determine if there is at least one responder after an associative search.

- Pick-One: Selects one responding responder from the set of responding PEs. It is implemented by ClearSpeed using GET and NEXT operations.

- Broadcast data or instructions from the control unit to PEs.

We have implemented these associative functions on the CSX600. To evaluate their running time, we store 10 records in each PE and perform each associative operation once for each of these 10 records. The timing results in microsecond ( $\mu$ sec or $10^{-6}$ second) are shown in Table I. Although they are not constant time, they are very efficient, and additionally establish that we can efficiently emulate an AP using ClearSpeed CSX600.

\section{AlgorithmS FOR ATC TASKS}

There are two basic components in our implementation, one is simulated radar reports in the host, and the other is tracks in PEs. Each radar report has its ID and 3D positions $\mathrm{X}, \mathrm{Y}$ and $\mathrm{H}$ etc. Each track has its ID, current 3D positions and velocities, predicted 3D positions and velocities, and positions of correlated report, etc. 


\section{A. Aircraft Tracking}

The aircraft tracking algorithm is shown in Algorithm 1. The input data are radar reports that are simulated in host and track records in PEs. If total time consumed is considered, this is easily the ATC task that consumes the most time, as it is performed much more frequently than the other tasks.

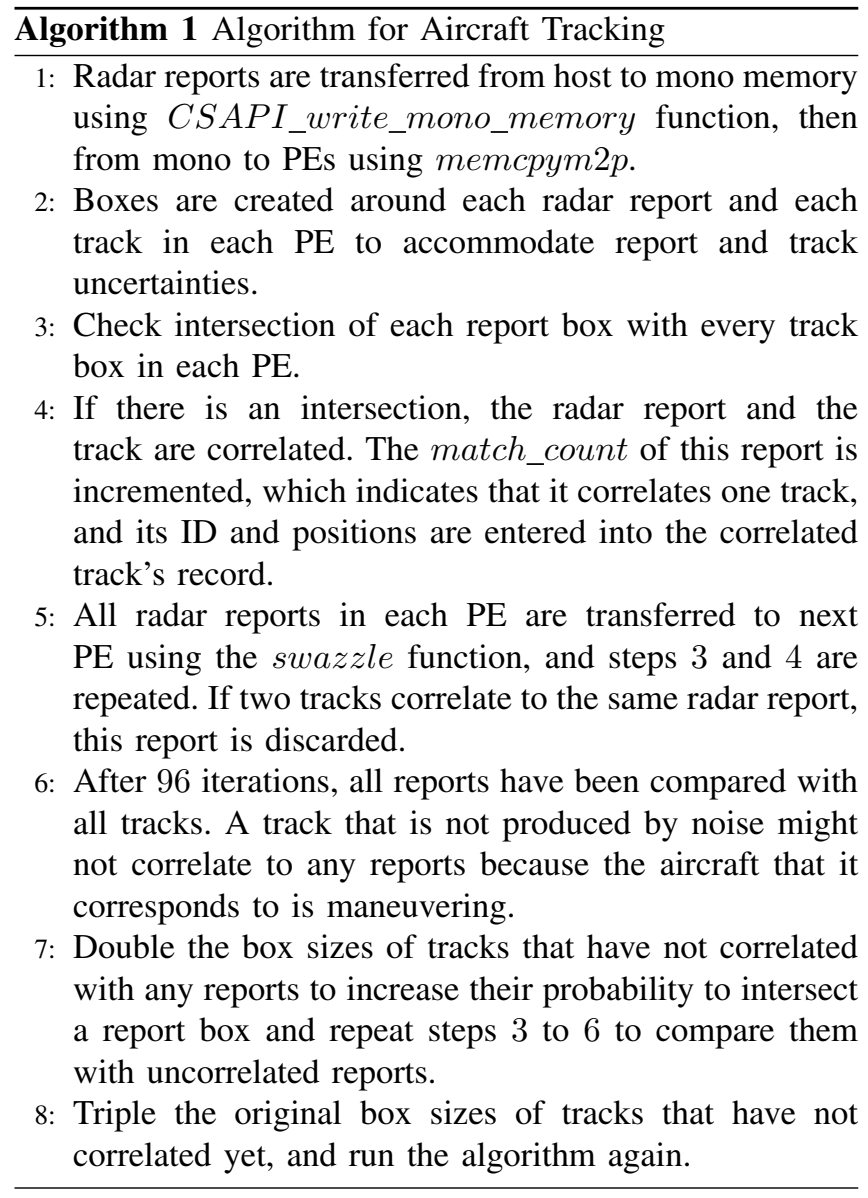

\section{B. Conflict Detection}

This paper considers a conflict to occur when two aircraft are predicted to be within a distance of three nautical miles in $\mathrm{x}$ and $\mathrm{y}$ and within 1000 feet in altitude. We assume that all aircraft to fly at the same altitude for simplicity. To assure timely evaluation we let the detection cycle be eight seconds, and we want to determine the possibility of a future conflict between any pairs of aircraft within a twenty minute "look ahead" period (i.e., 1200 seconds). The conflict detection algorithm is shown in Algorithm 2. The input data are the track records in PEs. We copy each track's $I D, 3 \mathrm{D}$ position $X_{t}, Y_{t}$ and $H_{t}$, and velocity $V_{x t}$ and $V_{y t}$ to the following variables, respectively, $I D, X_{c}, Y_{c}, H_{c}, V_{x c}$ and $V_{y c}$, to a poly structure collision in PEs. Initialize their time_till which is the aircraft's earliest collision time with other aircraft to 1200.00 .
Algorithm 2 Algorithm for Conflict Detection

1: For each collision and track record in each PE, first check whether their flight $I D s$ are different and altitudes are within 1000 feet.

2: Project their positions into 20 minutes, add 1.5 to each $x$ and $y$ edge of the future position to provide a 3.0 minimum miss distance, which is shown in Figure 3.

3: Calculate the min $\_x, \max \_x, \min \_y$ and $\max \_y$ for minimum and maximum intersection times in $x$ and $y$ dimensions, which are illustrated in equations $1,2,3$ and 4.

4: Find the largest minimum time time_min and smallest maximum time time_max across the two dimensions using equations 5 and 6 .

5: If time_min is less than time_max, there is a potential conflict between the aircraft whose ID is collision.ID and another aircraft whose ID is track.ID.

6: If time_min is less than collision.time_till, collision.time_till is updated to time_min.

7: All collision records in each PE are passed to next PE by swazzle function and steps 1 to 6 are repeated.

8: After 96 iterations, all collisions have been compared with all tracks. The time_till of each collision is its soonest collision time with another track.

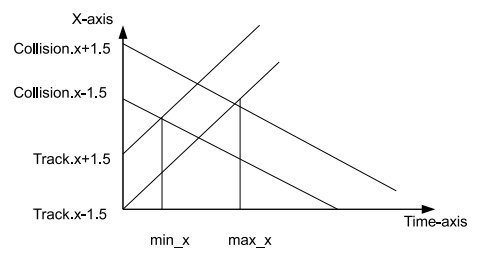

Figure 3: Conflict detection

The following formulas are used in Algorithm 2.

$$
\begin{aligned}
& \min \_x=\frac{\mid \text { collision. } X_{c}-\operatorname{track} . X_{t} \mid-3}{\left|\operatorname{collision} . V_{x c}-\operatorname{track} . V_{x t}\right|} \\
& \max \_x=\frac{\mid \text { collision. } X_{c}-\operatorname{track} . X_{t} \mid+3}{\left|\operatorname{collision} . V_{x c}-\operatorname{track} . V_{x t}\right|} \\
& \text { min_y }=\frac{\left.\mid \text { collision. } Y_{c}-\operatorname{track} . Y_{t}\right) \mid-3}{\mid \text { collision. } V_{y c}-\operatorname{track} . V_{y t} \mid} \\
& \max \_y=\frac{\left.\mid \operatorname{collision} . Y_{c}-\operatorname{track} . Y_{t}\right) \mid+3}{\left|\operatorname{collision} . V_{y c}-\operatorname{track} . V_{y t}\right|} \\
& \text { time_min }=\max \left\{\min \_x, \min \_y\right\} \\
& \text { time } \_\max =\min \left\{\max \_x, \max \_y\right\}
\end{aligned}
$$




\section{Conflict Resolution}

The algorithm for conflict resolution is described in the Algorithm 3. The input data are the tracks and collision records in PEs. Each PE can have 1 to 17 tracks and collision records.

\section{Further Discussion of ATC Algorithms}

Because there is only $6 \mathrm{~K}$ memory in each PE in CSX600, it is impossible to store unlimited tracks in one PE. At most 17 tracks can be assigned to each PE in the current implementation, so a maximum of 1632 tracks are stored in the PEs. When we process more than 1632 tracks, we store them in mono and transfer 1632 tracks to PEs each iteration until all have been processed. For example, it takes ten iterations to process 16320 tracks. To our surprise, the data transfer between mono and PEs only takes very few cycles, while the comparison, addition, subtraction and multiplication etc operations take many more cycles. We assume that it is due to CSX600 architecture.

\section{EXPERIMENTAL RESULTS}

This section describes the results of a set of preliminary experiments that were conducted to achieve three different goals. First, the experiments provide a proof-of-concept for the proposed ATC system implementation based on the ClearSpeed CSX600 hardware. Next, the performance and scalability of the proposed approach will be evaluated by performing a preliminary comparison between the ClearSpeed CSX600-based implementation of the tracking and correlation task, and two alternative implementations, namely a single-threaded version and a multi-threaded version running on a state-of-the-art multiprocessor/multicore server system featuring a total of 8 system cores. Finally, the experiments will provide some initial evidence for the claim that the proposed SIMD-based ATC system implementation exhibits a significantly larger degree of predictability than the MIMD-based solution.

\section{A. Experimental Setup}

Since we are creating a prototype solution, our implementations cannot manage the number of aircraft in a realworld situation. In order to have information about flights that we can control, we simulate the real-world situation by generating aircraft flights in a two dimensional airspace of 1024 by 1024 nautical miles. The initial positions and velocities of the aircraft are generated randomly and trajectories of aircraft consist of a constant velocity mode and a coordinated turn mode. Some radar noise is randomly generated. Since we control this process, we can generate different numbers of aircraft to test algorithms and test the limits on the number of aircraft that can be processed fast enough to meet deadlines. Unlike live flight data, when two aircraft are on a collision course, we can alter the flight path of one aircraft to eliminate this problem.
Algorithm 3 Algorithm for Conflict Resolution

1: Find the minimum time_till of all collisions records sequentially in each PE.

2: Find the minimum time_till across PEs using associative operation cs_reduce_min.

3: Transfer the collision records from PEs to mono using associative operation memcpyp $2 m$.

4: Search for the ID of the aircraft that has minimum time_till from the first aircraft to the end in mono sequentially because step 2 can only find the minimum time_till but cannot record its ID. This is the best or trial aircraft that will make the heading change.

5: Calculate that aircraft's velocity and angle using its velocity in $x$ and $y$ dimension in the mono memory.

6: We have projectedpath[96] in mono, copy the best aircraft's $I D$ and positions to all of the records of projectedpath [96], initialize all of their soonest collision time with other aircraft time_till to 1200.00 .

7: Each of the projectedpath $[i]$ represents a path where the best aircraft makes a different heading change from left to right 3 degrees to evaluate numerous different possible paths for the trial aircraft in parallel.

8: Transfer the projectedpath $[0], \cdots$,projectedpath [96] from mono to PEs each of which has one projectedpath $[i]$ using ClearSpeed associative operation memcpym $2 p$.

9: Each projectedpath is compared to all collision records in each PE: if their flight $I D s$ are different (so that the trial aircraft will not compare to itself) and altitudes are within 1000 feet, calculate $\min \_x, \max \_x, \min \_y$ and max $\_y$ for minimum and maximum intersection times in $x$ and $y$ dimension using the same equations $1,2,3$ and 4 .

10: Use equations 5 and 6 to get time_min and time_max. If time_min is less than time_max, there is a potential conflict between the aircraft whose $I D$ is the collision $I D$ and this path.

11: Check whether time_min is less than the time_till of this projectedpath, if so, this projectedpath.time_till is updated to time_min.

12: The projectedpath records in each PE are then passed to the next PE using swazzle function to compare with collision records in the neighbor PE.

13: After 96 iterations, all projectedpath records have been compared to all collision records, i.e., all other aircraft.

14: Find the maximum time_till using associative function cs_reduce_max because there is only one projectedpath.time_till record in each PE.

15: Transfer projectedpath records from PEs to mono using associative function memcpyp $2 m$.

16: Find which new path has the maximum time_till. This path is the best scenario. Record the $I D$ of it as minid.

17: Transfer all track records in each PE to mono using associative function memcpyp $2 m$.

18: The track whose $I D$ is minid is the best aircraft, change its $x$ and $y$ velocity to the best scenario path's velocity.

19: Display the resolution advisory and change the flight plan in the host. 
The SIMD-based implementation is based on the ClearSpeed CSX600 board and implemented in the $C^{n}$ language as described in the previous section II. Note that only one of the two CSX600 chips, i.e., one MTAP with a total of 96 PEs, has been used for the experiments. The reason for this is that with the CSX600, the two chips cooperate as two separate SIMDs. The latest SDK CSX600 software makes it easier to have the two chips operate as a single SIMD, but is still not as good a simulation of SIMD as obtained by using only one single chip.

The alternative implementations were all executed on an Intel Dual Processor Xeon E5410 Quad Core $2.33 \mathrm{GHz}$ system with $32 \mathrm{~GB}$ of main memory and $2 \times 6 \mathrm{Mb}$ of L2 cache (for each CPU). The systems feature a total of 8 cores. The operating system environment consists of Ubuntu 64-bit Linux kernel release 2.6.22-14-generic. The implementation was done in $\mathrm{C}$ using the gec compiler version 4.1.3. We used the compiler optimization flags '-mfpmath=387, sse msse3 -ffast-math -O3' to enable Intel's Streaming SIMD Extensions (SSE) for both the single-threaded as well as the multi-threaded version of the code. The single-threaded version is executed on a single core. The multi-threaded version is based on POSIX PThreads. It was carefully designed to minimize typical performance limiting effects such as false sharing, cache-ping-pong, and high lock contention. We specifically designed the multi-threaded code in such a way that locking was avoided whenever possible.

\section{B. Experiment Results}

First, we measure the timings in pure SIMD environment of CSX600. Our current implementation allows a maximum of 17 tracks per PE, i.e., 1632 tracks in total. The results are shown in Figure 4 and Figure 5: the horizontal axis represents the number of tracks and the vertical axis the execution time of the tasks in seconds. We assess the scalability of the tracking and CD\&R algorithms by evaluating their running time over a wide range of number of tracks and plot the time each algorithm takes on a graph. We can see that the CSX approach can finish all the three tasks within deadlines even when the number of tracks is scaled up to 1500 .

The sequential complexity of both algorithms illustrated in Figures 4 and 5 are $O\left(n^{2}\right)$, because each report box is evaluated with each track box. The CSX can execute 96 instances of a task in essentially the same time as it requires to execute 1 instance of this task. However, 96 is not a constant with respect to $n=1632$, as $\log 1632$ is about 10.7 and 1632 is roughly 96 raised to the 1.6 power. Since the sequential complexity of both algorithms is quadratic, the runtime of the CSX600 on both of these algorithms should be faster than quadratic but slower than linear. This faster performance of the CSX600 is particularly evident in Figure 6.

Second, the tracking and correlation was performed under all three approaches. In the following we will also refer

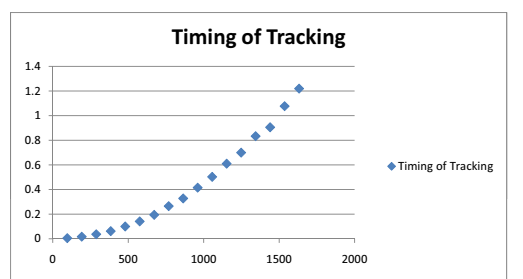

Figure 4: Timing of Tracking Algorithm

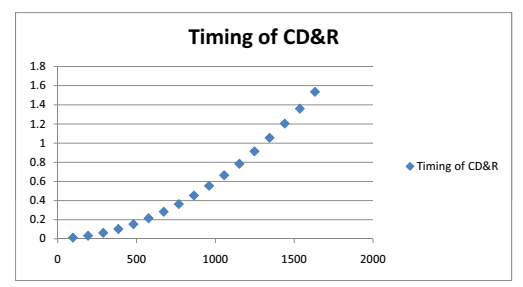

Figure 5: Timing for CD\&R Algorithm

to the ClearSpeed-based ATC implementation as SIMD, to the single-threaded implementation as $S T I$, and to the multi-threaded implementation as $M T I$. Each approach was executed for a varying number of planes, ranging from 4000 to 14000 in increments of 1000 . For each approach, and for each number of planes in the given range, each approach was executed for 50 iterations. One iteration corresponds to one radar refresh cycle. Hence, a total of 50 sets of incoming radar reports had to be completed. We measured the execution time for each iteration. Note that since none of these approaches ever idles to wait for new incoming radar reports, the number of iterations divided by the total execution time represents the highest-possible radar update rate that can be sustained by each approach.

Figure 6 shows the maximum value of the execution times for each of the experiments and for each of the three approaches. From these results we can see that $S T I$ always takes the most time and increases the quickest. $M T I$ takes a little less time than $S I M D$ from 4000 to 7000 aircraft, their time is similar between 7000 and 8000 aircraft, and from 8000 aircraft and on, MTI takes more time and increases quicker than $S I M D$. In addition, the performance results on the CSX600 portrays a linear time per aircraft shown in Figure 6 because as mentioned in Section IV-D, 1632 tracks are processed each time, so it takes nine iterations to process 14688 tracks as our worst case, and the data transfer takes very short time while computation takes most of the time.

While the results in Figure 6 demonstrate the performance benefits of $S I M D$, Figure 7 focuses on the predictability of the execution times, which is an important factor in guaranteeing that all the ATC processing can be performed within the specified time bounds. The result in Figure 7 measure the Coefficient of Variation (COV), which is a common normalized measure of dispersion, and is defined 


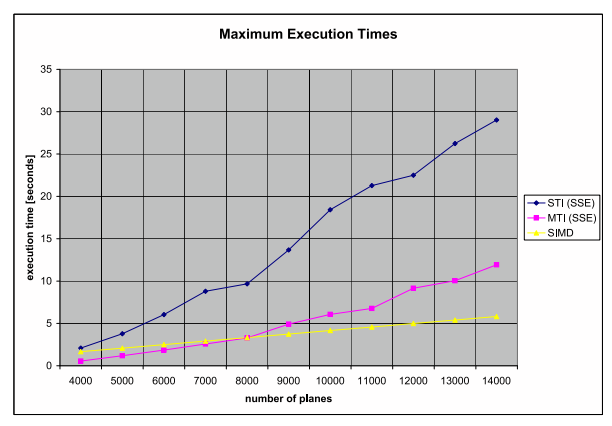

Figure 6: Performance of $S T I, M T I$, and $S I M D$

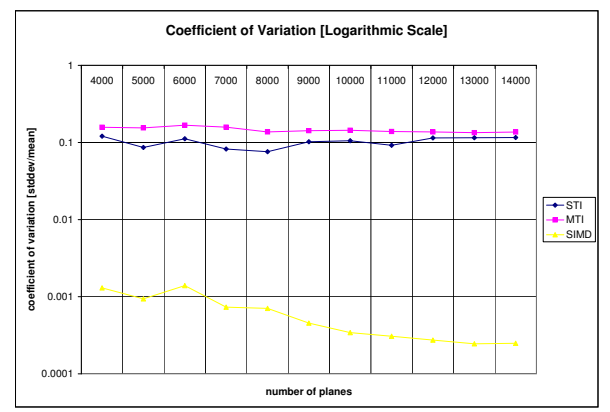

Figure 7: Predictability of Execution Times for $S T I, M T I$, and $S I M D$

as the ratio of the standard deviation to the mean. Unlike the standard deviation, the $\mathrm{COV}$ is dimensionless. Note that the $y$-axis in Figure 7 uses a logarithmic scale. The results clearly show that the COV values for SIMD are several orders of magnitude below the ones for STI and MTI. We attribute the fluctuations in the execution times of both STI and MTI to both operating system and hardware interference.

\section{CONClusions And Future Work}

In this paper, we propose an efficient and scalable solution for Air Traffic Control problems using the associative processor system that is emulated on the ClearSpeed CSX600 system. The contributions of this paper are summarized as follows. First, the AP system proposed can not only estimate aircraft states, detect conflicts, and resolve conflicts accurately, but also process large numbers of aircraft within deadlines. Because the emulation tool CSX600 can only process maximum 1500 aircraft within deadline in Section V-B and our current prototype involves only three ATC tasks, an ideal AP system would have to be a lot larger than the CSX600. As discussed earlier, the ideal AP should have at least 14000 processors in order to provide reasonable flexibility in the number of distinct ATC tasks executed and to meet all worst case execution deadlines for each of these tasks. Since all PEs would be assigned at most one aircraft, this system should provide roughly a linear speedup over sequential performance. Moreover, the worst case running time for the proposed AP system could be accurately predicted. In contrast, the MIMD-based systems are evaluated only on average running time and have highly unpredictable worst case running times. These two contributions can provide major help in meeting the goals of FAA's NextGen Plan: fly more aircraft, more safely, more precisely, more efficiently and use less fuel [31]. Third, the software used by AP will be substantially simpler and smaller in size than the current corresponding ATC software. Fourth, the V\&V (Validation and Verification) process is simpler than that for current ATC software. Fifth, the hardware architecture of AP is simpler than the current ATC MIMD hardware. Moreover, considering the AP's advantages of simple programming style and simple hardware implementations, it obviously deserves more attention and utilization if we want to obtain satisfactory solutions to numerous critical real-time problems with hard deadlines including ATC.

In the future, we will complete implementing additional algorithms for the basic ATC tasks on ClearSpeed and compare this system's performance with the performance of MIMD systems of similar power, as in Section V. A possibly important extension to our current research would be to consider a GPU implementation using CUDA. Nvidia has many SIMD PE groups on its chips. The Nvidia technology including the latest FERMI chip has a lot in common with the MTAP approach of ClearSpeed, and implementing the CSX600 ATC algorithms on this architecture may provide another useful platform to use in this project.

\section{ACKNOWLEDGMENTS}

We wish to thank anonymous reviewers for their valuable comments, which were very useful in the production of the final version of this paper.

\section{REFERENCES}

[1] Y. Bar-Shalom and T.E. Fortmann, Tracking and Data Association. Academic Press, 1988.

[2] Y. Bar-Shalom and X.R. Li, Estimation and Tracking: Principles, Techniques and Software. Artech House, Boston, 1993.

[3] H. Blom and Y. Bar-Sharlom, The interacting multiple model algorithm for systems with markovian switching coefficients. IEEE Transactions on Automatic Control, 33(8):780-783, August 1988.

[4] H.A.P. Blom, R.A. Hogendoorn, and B.A. van Doorn, Design of a multisensor tracking system for advanced air traffic control. In Y. Bar-Shalom, editor, Multitarget-Multisensor Tracking: Application and Advances, volume 2, pages 31-63, Artech House, 1990.

[5] Y-J. Chiang, J. T. Klosowski, C. Lee and J. S. B. Mitchell, Geometric Algorithms for Conflict Detection/Resolution in Air Traffic Management, 36th IEEE Conference on Decision and Control, pages 1835-1840, San Diego, CA, December 1997. 
[6] M. Garey and D. Johnson, Computers and Intractability: a Guide to the Theory of NP-completeness. W.H. Freeman, 6566, 238-240, New York, 1979.

[7] J.L. Gustafson and B.S. Greer. ClearSpeed whitepaper: accelerating the Intel Math Kernel Library. http://www.clearspeed.com/docs/resources/ClearSpeed Intel Whitepaper Feb07.pdf, 2007.

[8] I. Hwang and C. Tomlin, Protocol-based Conflict Resolution for Finite Information Horizon. Proceedings of the AACC American Control Conference, Anchorage, May 2002.

[9] I. Hwang, J. Hwang, and C. Tomlin, Flight-Mode-Based Aircraft Conflict Detection using a Residual-Mean Interacting Multiple Model Algorithm. Proceedings of the AIAA Guidance, Navigation, and Control Conference, Austin Texas, August 2003.

[10] I. Hwang, H. Balakrishnan, K. Roy, and C. Tomlin, MultipleTarget Tracking and Identity Management in Clutter for Air Traffic Control. Proceedings of the AACC American Control Conference, Boston, MA, June 2004.

[11] M. Jin, J. Baker and K. Batcher, Timings for Associative Operations on the MASC Model. Proc. of the 15th International Parallel and Distributed Processing Symposium (IEEE Workshop on Massively Parallel Processing), San Francisco, 2001 .

[12] S. Kahne and I. Frolow, Air traffic management: Evolution with technology. IEEE Control Systems Magazine, 16(4):1221, 1996

[13] J. Krozel, M. Peters, K.D. Bilimoria, C. Lee, and J.S.B. Mitchell, System Performance Characteristics of Centralized and Decentralized Air Traffic Separation Strategies. Fourth USA/Europe Air Traffic Management Research and Development Seminar, 2001.

[14] J.K. Kuchar and L.C. Yang, A review of conflict detection and resolution modeling methods. IEEE Transactions on Intelligent Transportation Systems, 1(4):179-189, 2000.

[15] D.G. Lainiotis, Partitioning: A unifying framework for adaptive systems I: Estimation. Proceedings of the IEEE, 64:11261142, August 1976.

[16] X.R. Li and Y. Bar-Shalom, Design of an interacting multiple model algorithm for air traffic control tracking. IEEE Transactions on Control Systems Technology, 1(3):186-194, September 1993.

[17] K.M. Liu, Composition of Kalman and heuristic tracking algorithms for air traffic control, master thesis, Kent State University, August 1999.

[18] E. Mazor, A. Averbuch, Y. Bar-Shalom, and J. Dayan, Interacting multiple model methods in tracking: A survey. IEEE Transactions on Aerospace and Electronic Systems, 34(1):103-123, 1998.

[19] W. Meilander, M. Jin, and J. Baker, Tractable Real-Time Air Traffic Control Automation. Proc. of the 14th IASTED International Conference on Parallel and Distributed Computing and Systems, 483-488, Cambridge, MA, 2002.
[20] W. Meilander, J. Baker, M. Jin, Predictable Real-Time Scheduling for Air Traffic Control. Fifteenth International Conference on Systems Engineering, 533-539, August 2002.

[21] W. Meilander, J. Baker, and M. Jin, Importance of SIMD Computation Reconsidered. Proc. of the 17th International Parallel and Distributed Processing Symposium (IEEE Workshop on Massively Parallel Processing), Nice, France, April 2003.

[22] M.S. Nolan, Fundamentals of Air Traffic Control. Brooks/Cole, Wadsworth, 3rd edition, 1998.

[23] R.A. Paielli and H. Erzberger, Conflict probability estimation for free flight. Journal of Guidance, Control, andDynamics, 20(3):588-596, 1997.

[24] J. Potter, J. Baker, S. Scott, A. Bansal, C. Leangsuksun, and C. Asthagiri, ASC: An Associative-Computing Paradigm, Computer, 27(11):19-25, 1994.

[25] M. Prandini, J. Hu, J. Lygeros and S. Sastry, A probabilistic Approach to Aircraft Conflict Detection, IEEE Transactions on Intelligent Transportation Systems, 1(4):199-219, December 2000

[26] S. Reddaway, W. Meilander, J. Baker, and J. Kidman, Overview of Air Traffic Control Using an SIMD COTS System. Proc. of the International Parallel and Distributed Processing Symposium (IPDPS'05), Denver, April 2005.

[27] J. Stankovic, M. Spuri, M. Natale and G. Buttazzo, Implications of Classical Scheduling Results for Real-Time Systems. IEEE Computer, 16-25, June 1995.

[28] D.D. Sworder and J.E. Boyd, Estimation Problems in Hybrid Systems. Cambridge University Press, 1999.

[29] L.C. Yang and J.K. Kuchar, Prototype conflict alerting system for free flight. Journal of Guidance, Control, and Dynamics, 20(4), July-August 1997.

[30] M.Yuan, J.W.Baker, F.Drews and W.Meilander, Efficient Implementation of Air Traffic Control Using the ClearSpeed CSX620 System. Proc. of the 21st IASTED International Conference on Parallel and Distributed Computing and Systems (PDCS), 353-360, Cambridge, MA, November, 2009.

[31] FAA's NextGen Implementation Plan, http://www.faa.gov/about/initiatives/nextgen/media/ngip.pdf, 2009.

[32] FAA Grants for Aviation Research Program Solicitation, http://www.tc.faa.gov/logistics/grants/, 2009.

[33] ClearSpeed Technology plc. ClearSpeed whitepaper: CSX processor architecture, http://www.clearspeed.com/docs/resources/, 2007.

[34] ClearSpeed Technology plc. ClearSpeed whitepaper: ClearSpeed Software Description, https://support.clearspeed.com/documents/, 2007.

[35] ClearSpeed Technology plc. CSX600 Runtime Software User Guide, https://support.clearspeed.com/documents/, 2007. 